SFB

Cointegration in singular 823 ARMA models

Manfred Deistler, Martin Wagner

Nr. 5/2017

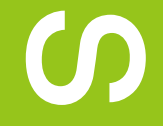

$\mathcal{O}$

(

$\overbrace{}^{2}$

(1)

(D)

(

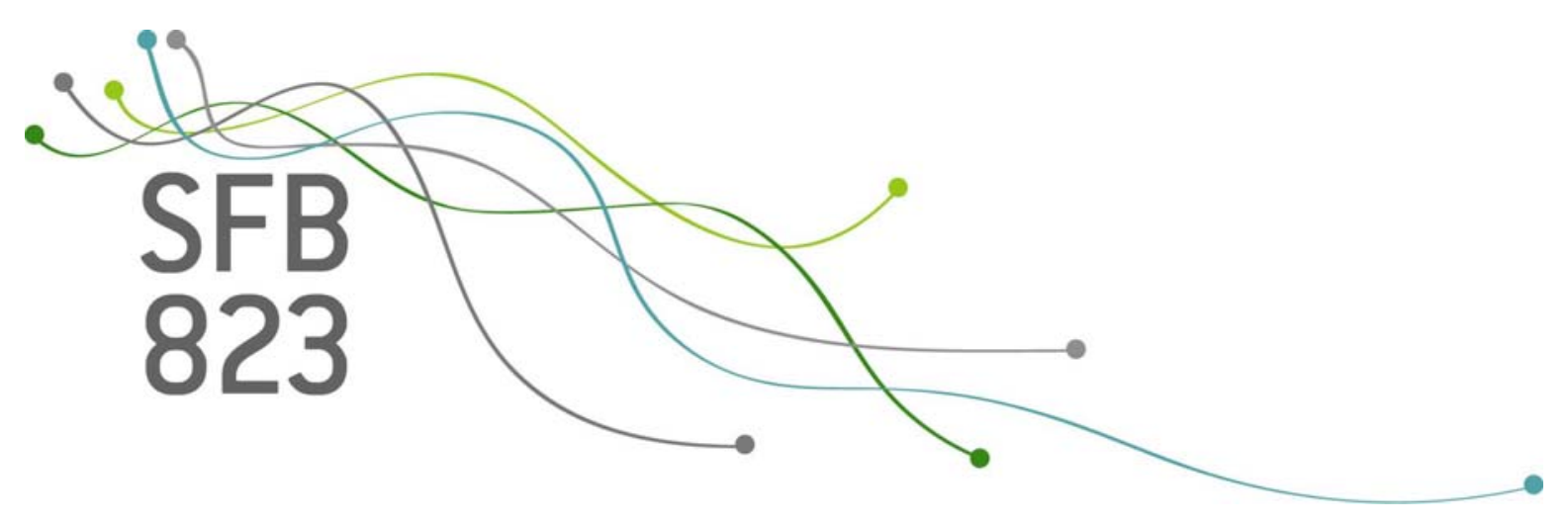





\section{Cointegration in Singular ARMA Models}

\author{
Manfred Deistler \\ Institute of Statistics and Mathematical \\ Methods in Economics \\ Technische Universität Wien \\ Vienna, Austria
}

Martin Wagner

Faculty of Statistics

Technical University Dortmund

Dortmund, Germany

\&

Institute for Advanced Studies

Vienna, Austria

\&

Bank of Slovenia

Ljubljana, Slovenia 
Proposed running head:

\section{Cointegration in Singular ARMA Models}

Corresponding author:

Martin Wagner

Faculty of Statistics

Technical University Dortmund

Vogelpothsweg 87

D-44227 Dortmund, Germany

Tel: $++49+2317553174$

Email: mwagner@statistik.tu-dortmund.de 


\begin{abstract}
We consider the cointegration properties of singular ARMA processes integrated of order one. Such processes are necessarily cointegrated as opposed to the regular case. We show that in the left coprime case the cointegrating space only depends upon the autoregressive polynomial at one.
\end{abstract}

JEL Classification: C32, C38

Keywords: Cointegration, Singular ARMA Systems 


\section{Introduction}

In this paper we analyze the (integration and) cointegration properties of singular AR and ARMA models. Singular AR and ARMA models, i.e., models with singular error variance, occur in the dynamic stochastic general equilibrium (DSGE) literature, if the number of shocks is strictly smaller than the number of observables (see, e.g., Komunjer and Ng, 2011). They also occur in linear dynamic factor models. In this context the latent variables are typically described as a singular AR or ARMA models (see, e.g., Forni et al., 2000). Furthermore, in this setting singular ARMA models may arise as models for the static factors, if the number of the static factors is strictly larger than the number of dynamic factors. In a stationary setting singular AR models are treated in Anderson et al. (2012) and the ARMA case is considered in Anderson et al. (2016).

Cointegration properties of singular AR models are analyzed in Barigozzi et al. (2016). Whereas regular AR systems are always left coprime, this is not true for singular AR systems, see Anderson et al. (2012), which thus show a similarity to ARMA models. Taking this similarity into account we are led to considering the cointegration properties of singular ARMA models. We first discuss that in the singular case cointegration is inevitably present. Then we show that in the left coprime case the cointegrating space only depends on the autoregressive polynomial at $z=1$, as in the regular case.

The paper is organized as follows: Section 2 introduces the setting and states the underlying assumption, Section 3 gives the result and Section 4 briefly concludes.

\section{Setting and Assumptions}

Consider an ARMA system

$$
a(z) y_{t}=b(z) \varepsilon_{t}
$$

where

$$
\begin{aligned}
& a(z):=\sum_{j=0}^{p} a_{j} z^{j}, a_{j} \in \mathbb{R}^{n \times n}, a_{0}=I_{n}, \\
& b(z):=\sum_{j=0}^{q} b_{j} z^{j}, b_{j} \in \mathbb{R}^{n \times q}
\end{aligned}
$$


with $z$ used as both a complex variable as well as the backward shift operator on the integers $\mathbb{Z}$. The process $\left\{\varepsilon_{t}\right\}$ is white noise with $\mathbb{E} \varepsilon_{t} \varepsilon_{t}=I_{q}$. The system is singular if $q<n$ and regular for $q=n$ and $\operatorname{rk} b(z)=q$ for one $z$.

Assumption 1 Throughout we assume that:

(i) The determinant of the AR polynomial fulfills

$$
\operatorname{det} a(z) \neq 0 \text { for }|z| \leq 1 \text {, except for } z=1 \text {. }
$$

(ii) System (1) fulfills the strict miniphase assumption

$$
r k b(z)=q \quad|z| \leq 1
$$

(iii) In addition we assume that the system (1) is integrated of order 1, i.e., the transfer function

$$
k(z):=a^{-1}(z) b(z)
$$

has a pole at $z=1$, but the function $c(z)=(1-z) k(z)$ has all poles outside the closed unit circle. Thus,

$$
c(z)=\sum_{j=0}^{\infty} c_{j} z^{j}
$$

is a convergent power series for $|z| \leq 1$.

(iv) The pair $(a(z), b(z))$ is left coprime, i.e., every common left (polynomial matrix) divisor of $a(z)$ and $b(z)$ is a unimodular matrix.

With respect to item (iv) of Assumption 1 note that a polynomial matrix $u(z)$ is unimodular if and only if $\operatorname{det} u(z) \equiv c \neq 0$ and that $(a(z), b(z))$ is left coprime if and only if $\operatorname{rk}(a(z), b(z))=n$ for all $z \in \mathbb{C}$. For a more detailed discussion see, e.g., Hannan and Deistler (2012).

\section{The Cointegration Properties in the Singular Case}

Let

$$
\begin{aligned}
k(z) & =a^{-1}(z) b(z) \\
& =u(z) \Lambda(z) v(z)
\end{aligned}
$$


denote the Smith-McMillan form (see, e.g., Hannan and Deistler, 2012) of the transfer function $k(z)$. Here $u(z)$ and $v(z)$ are unimodular $n \times n$ respectively $q \times q$ matrices and $\Lambda(z)$ is a unique $n \times q$ diagonal matrix of the form

$$
\Lambda(z):=\left(\begin{array}{ccc}
\frac{p_{1}(z)}{q_{1}(z)} & & 0 \\
& \ddots & \\
& & \frac{p_{q}(z)}{q_{q}(z)} \\
\hline 0 & \cdots & 0
\end{array}\right),
$$

where $p_{i}(z), q_{i}(z)$, for $i=1, \ldots, q$ are relatively prime and monic (i.e., with leading coefficient equal to one) polynomials, $p_{i}(z)$ divides $p_{i+1}(z)$ for $i=1, \ldots, q-1$ and $q_{i+1}(z)$ divides $q_{i}(z)$ for $i=1, \ldots, q-1$. For a given transfer function $k(z)$, the zeros of the polynomials $p_{i}(z)$ are the zeros of $k(z)$ and the zeros of the polynomials $q_{i}(z)$ are the poles of $k(z)$.

The strict miniphase assumption (3) implies that $p_{i}(1) \neq 0$ for $i=1, \ldots, q$ and condition (2) implies that the zeros of $q_{i}(z)$ are either at $z=1$ or satisfy $|z|>1$. Consequently, there is a $c$, with $1 \leq c \leq q$, such that

$$
q_{i}(z)=(1-z) \bar{q}_{i}(z), \quad i=1, \ldots, c
$$

with $\bar{q}_{i}(1) \neq 0$ for $i=1, \ldots, c$ and $q_{i}(1) \neq 0$ for $i=c+1, \ldots, q$. Accordingly we may write

$$
\Lambda(z)=\left(\begin{array}{cccccc}
\frac{p_{1}(z)}{\overline{q_{1}(z)}}(1-z)^{-1} & 0 & & \ldots & & 0 \\
0 & \ddots & \ddots & & 0 \\
& \ddots & \frac{p_{c}(z)}{\bar{q}_{c}(z)}(1-z)^{-1} & & & \vdots \\
\vdots & & & \frac{p_{c+1}(z)}{q_{c+1}(z)} & & \\
& & \ddots & & \ddots & 0 \\
0 & & \cdots & 0 & & \frac{p_{q}(z)}{q_{q}(z)} \\
\hline 0 & \cdots & \cdots & \cdots & \cdots & 0
\end{array}\right),
$$

and

$$
c(z)=u(z)\left(\begin{array}{cccccc}
\frac{p_{1}(z)}{\bar{q}_{1}(z)} & 0 & & \cdots & & 0 \\
0 & \ddots & \ddots & & & 0 \\
& \ddots & \frac{p_{c}(z)}{\bar{q}_{c}(z)} & & & \vdots \\
\vdots & & & \frac{p_{c+1}(z)}{q_{c+1}(z)}(1-z) & & \\
& & & & \ddots & 0 \\
0 & & \cdots & 0 & & \frac{p_{q}(z)}{q_{q}(z)}(1-z) \\
\hline 0 & \cdots & \cdots & \cdots & \cdots & 0
\end{array}\right) v(z)
$$


Now, consider, a Beveridge-Nelson type decomposition (see, e.g., Beveridge and Nelson, 1981; Phillips and Solo, 1992)

$$
c(z)=c(1)+(1-z) c^{*}(z)
$$

with $c^{*}(z)$ rational with no poles and zeros inside or on the unit circle.

The solution of the ARMA system on $\mathbb{N}$ we consider (see, e.g., Bauer and Wagner, 2012) is of the form

$$
y_{t}=c(1) \sum_{j=1}^{t} \varepsilon_{j}+c^{*}(z) \varepsilon_{t} .
$$

Taking the first difference of $y_{t}$ as given in 12 leads to a process $y_{t}-y_{t-1}=\sum_{j=0}^{\infty} c_{j} \varepsilon_{t-j}$ for $t \geq 2$ that is stationary. As is immediately clear from (12), the left kernel of $c(1)$, lker $c(1)$, is the space of cointegrating relationships $\beta$, say. Clearly, the cointegrating space has at least dimension $n-q$. Thus, in the singular case cointegration is always present. Since $\frac{p_{i}(1)}{\bar{q}_{i}(1)} \neq 0$ and both $u(1)$ and $v(1)$ are nonsingular, it is directly seen from the that the dimension of lker $c(1)$ is equal to $n-c$.

Defining

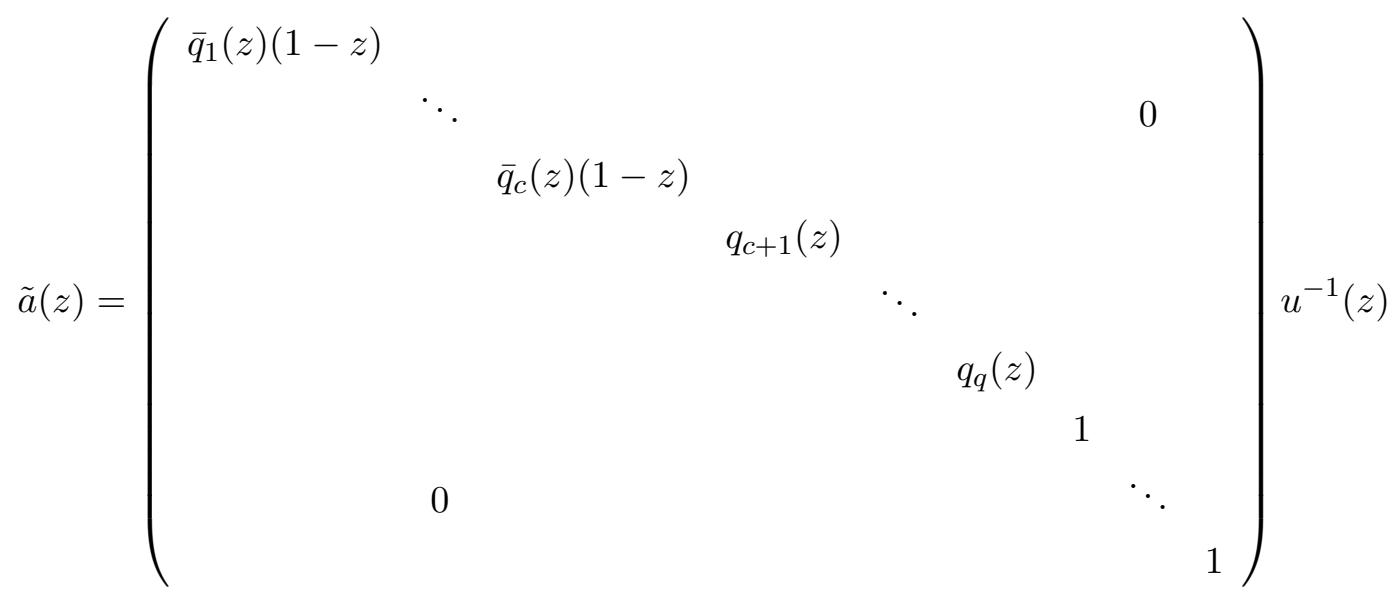

and

$$
\tilde{b}(z)=\left(\begin{array}{ccc}
p_{1}(z) & & 0 \\
& \ddots & \\
0 & & p_{q}(z) \\
\hline 0 & \cdots & 0
\end{array}\right) v(z)
$$

it holds that $k(z)=\tilde{a}^{-1}(z) \tilde{b}(z)$ and thus $(\tilde{a}(z), \tilde{b}(z))$ is an ARMA system corresponding to $k(z)$. As can easily be shown from the properties of the Smith-McMillan form $(\tilde{a}(z), \tilde{b}(z))$ is left coprime. 
From (13) we see that the left kernel of $\tilde{a}(1)$ has dimension $c$, and as every left coprime pair $(a(z), b(z))$ corresponding to $k(z)$ is related to $(\tilde{a}(z), \tilde{b}(z))$ via unimodular left multiplication (compare again Hannan and Deistler, 2012), this is true for all left coprime ARMA systems $(a(z), b(z))$ such that $k(z)=a^{-1}(z) b(z)$.

Now, from 10 and 13 we see

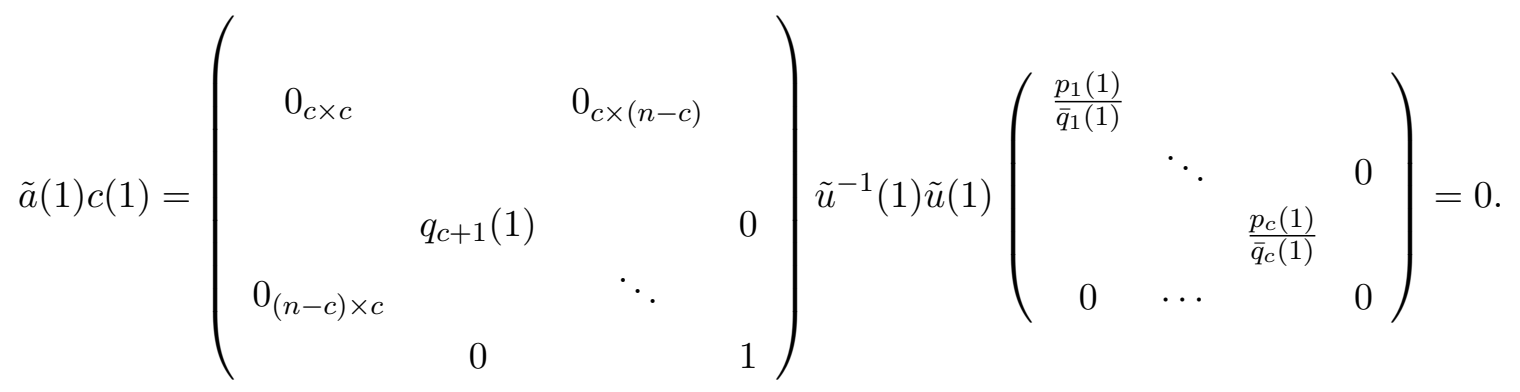

Summarizing the discussion we have shown the following:

Theorem 1 For every left coprime pair $(a(z), b(z))$ fulfilling Assumption 1 such that $k(z)=$ $a^{-1}(z) b(z)$, the rank of $a(1)$ is equal to the dimension of the cointegrating space for the process $y_{t}, t=1,2, \ldots$ given by (12). Moreover, the linearly independent non-zero rows of $\tilde{a}(1)$ span the ortho-complement of the cointegrating space.

Remark 1 In the non left coprime case, the dimension of the cointegrating space does not only depend upon a(1). For a discussion of this issue in the regular ARMA case see also Deistler and Wagner (2000).

Remark 2 Note that in the singular case, exact cointegration might be present, i.e., there could exist some (non-zero) vectors $\beta$, such that $\beta^{\prime} y_{t}=0$ for $t=1,2, \ldots$ This happens, compare again (12), if in addition to $\beta^{\prime} c(1)=0$ (corresponding to cointegration) also $\beta^{\prime} c^{*}(z)=0$ holds. Note first that this cannot happen in the regular case as $c^{*}(z)$ has by construction no zeros and poles on the unit circle and is thus almost everywhere nonsingular. This in turn implies that (rational) elements of the kernel have to be zero almost everywhere. In the singular case, however, $c^{*}(z)$ may contain, e.g., zero rows and nevertheless satisfy that its poles and zeros are outside the closed unit circle.

Remark 3 It is common in the autoregressive cointegration literature to consider the so-called Granger or Granger-Johansen representation (see, e.g., Johansen, 1995, Theorem 4.2). In this 
representation the matrix $c(1)$ is expressed in terms of the underlying AR or ARMA parameters. Thus, using standard notation, write $-a(1)=\alpha \beta^{\prime}$ with $\alpha, \beta \in \mathbb{R}^{n \times(n-c)}$ with full column rank and define $\alpha_{\perp}, \beta_{\perp} \in \mathbb{R}^{n \times c}$ to span the ortho-complements of the column spaces of $\alpha$ and $\beta$ respectively. Using this notation we can rewrite (12) as

$$
\begin{aligned}
y_{t} & =c(1) \sum_{j=1}^{t} \varepsilon_{j}+c^{*}(z) \varepsilon_{t} \\
& =\beta_{\perp}\left(\alpha_{\perp}^{\prime}\left(I_{n}-\sum_{i=1}^{p} a_{i}\right) \beta_{\perp}\right)^{-1} \alpha_{\perp}^{\prime} b(1) \sum_{j=1}^{t} \varepsilon_{j}+c^{*}(z) \varepsilon_{t} .
\end{aligned}
$$

The strict miniphase assumption implies that $b(1)$ has full column rank $q$ and left coprimeness implies that the left kernel of $c(1)$ is given by $\beta$. In the regular case, with $b(1)$ a regular $n \times n$ matrix, a similar result - the dependence of the cointegrating spaces on the autoregressive parameters only - has been shown in Deistler and Wagner (2000) for processes with arbitrary integration orders.

\section{Conclusion}

We have shown that singular ARMA processes integrated of order one are necessarily cointegrated. As in the regular case, left coprimeness of the ARMA representation guarantees that the cointegrating space only depends upon the autoregressive polynomial at one. Furthermore, we have noted that in the singular case exact cointegration may occur, i.e, there may be static linear combinations of the process that are identically zero. Note that our finding implies that in left coprime singular AR or ARMA representations of integrated processes of order one the number of error correction terms is unique. This implies that the non-uniqunesss of the number of error correction terms reported in Barigozzi et al. (2016) must be related to the consideration of non left coprime systems.

\section{Acknowledgements}

The first author acknowledges financial support from the Fonds zur Förderung der wissenschaftlichen Forschung (FWF) via project P24198/N18. The second author acknowledges financial support from the Deutsche Forschungsgemeinschaft via the Collaborative Research Center "Statistical Modelling of Nonlinear Dynamic Processes" (SFB 823). The usual disclaimer applies. 


\section{References}

Anderson, B.D.O., W. Chen, M. Deistler and A. Filler (2012). AR Models of Singular Spectral Densities. Automatica 48, 2843-2849.

Anderson, B.D.O., M. Deistler, E. Felsenstein and L. Koelbl (2016). The Structure of Multivariate AR and ARMA Systems: Regular and Singular Systems; the Single and the Mixed Frequency Case. Journal of Econometrics 192, 366-373.

Barigozzi, M., M. Lippi and M. Luciani (2016). Dynamic Factor Models, Cointegration, and Error Correction Mechanisms. Mimeo.

Bauer, D. and M. Wagner (2012). A State Space Canonical Form for Unit Root Processes. Econometric Theory 28, 1319-1349.

Beveridge, S. and C.R. Nelson (1981). A New Approach to Decomposition of Economic Time Series into Transitory and Permanent Components with Particular Attention to Measurement of the "Business Cycle". Journal of Monetary Economics 7, 151-174.

Deistler, M., A. Filler and B. Funovits (2011). AR Systems and AR Processes, the Singular Case. Communications in Information and Systems 11, 225-236.

Deistler, M. and M.Wagner (2000). On the Structure of Cointegration. In Dockner, E. et al. (Eds.): Optimization, Dynamics and Economic Analysis, Spinger, 373-379.

Forni, M., M. Hallin, M. Lippi and L. Reichlin (2000). The Generalized-Dynamic Factor Model: Identification and Estimation. Review of Economics and Statistics 82, 540-554.

Hannan, E.J. and M. Deistler (2012). The Statistical Theory of Linear Systems. Wiley, New York, 1988. Reprint in SIAM Classics in Applied Mathematics, Philadelphia.

Johansen, S. (1995). Likelihood-Based Inference in Cointegrated Vector Autoregressive Models. Oxford University Press, Oxford.

Komunjer, I. and S. Ng (2011). Dynamic Identification of Dynamic Stochastic General Equilibrium Models. Econometrica 79, 1995-2032.

Phillips, P.C.B. and V. Solo (1992). Asymptotics for Linear Processes. Annals of Statistics 20, $971-1001$. 

